\title{
CONTROL DE Euphorbia dentata EN SOJA CON HERBICIDAS POST-EMERGENTES
}

\author{
Víctor F. Juan', Horacio M. Saint-André2, Ricardo R. Fernández², \\ Martín Videla ${ }^{3}$ y Juan Bazzano ${ }^{3}$
}

\author{
${ }^{1}$ Ingeniero Agrónomo, MSc, Profesor. Facultad de Agronomia / UNICEN. Casilla 47. (7300) - Azul, Pcia. de Buenos Aires, Argentina. \\ vjuan@faa.unicen.edu.ar \\ ${ }^{2}$ Ingeniero Agrónomo, Profesor. Facultad de Agronomia / UNICEN.hsaint@faa.unicen.edu.ar , rrf@,faaunicen.edu.ar \\ ${ }^{3}$ Ayudante de Investigación. Facultad de Agronomia / UNICEN.
}

\begin{abstract}
RESUMEN
Euphorbia dentata es una maleza anual, de ciclo primavero estival, que ha adquirido importancia asociada principalmente al cultivo de soja en la zona Centro de la Provincia de Buenos Aires República Argentina. Esta es considerada una especie de difícil control y altamente competitiva del cultivo. El objetivo de este trabajo fue evaluar la eficacia de control de Euphorbia dentata con herbicidas post emergentes en el cultivo de soja. El cultivo fue implantado en el partido de Azul, sobre un lote con alta infestación de la maleza lográndose un stand homogéneo de 110 plantas $/ \mathrm{m}^{2}$. Sobre éste se realizaron los siguientes tratamientos: imazethapyr $100 \mathrm{~g} / \mathrm{ha}$, clorasulam-metil $33,6 \mathrm{~g} / \mathrm{ha}+$ coadyuvante no iónico $0,2 \% \mathrm{v} / \mathrm{v}$, lactofén $96 \mathrm{~g} / \mathrm{ha}+$ coadyuvante no iónico $0,2 \% \mathrm{v} / \mathrm{v}$, clorimurón-etil $8,75 \mathrm{~g} / \mathrm{ha}+$ coadyuvante no iónico $0,2 \% \mathrm{v} / \mathrm{v}$, clorasulam-metil $33,6 \mathrm{~g} / \mathrm{ha}+$ lactofen $96 \mathrm{~g} / \mathrm{ha}+$ coadyuvante no iónico $0,2 \% \mathrm{v} / \mathrm{v}$ y clorimurón-etil $8,75 \mathrm{~g} / \mathrm{ha}+$ lactofen $96 \mathrm{~g} / \mathrm{ha}+$ coadyuvante no iónico $0,2 \% \mathrm{v} / \mathrm{v}$, testigo enmalezado y testigo limpio. Se utilizó un diseño de bloques al azar con 4 repeticiones y se realizaron evaluaciones de control a los 20 y 45 días desde la aplicación y de rendimiento de soja a cosecha. Ninguno de los tratamientos ensayados alcanzó el $70 \%$ de control. Los máximos niveles de eficacia fueron obtenidos con imazethapyr (67\%) y con la mezcla de clorasulam+lactofen $(65 \%)$ que no presentaron diferencias significativas entre sí. El rendimiento del cultivo mostró una respuesta similar, siendo la productividad en esos tratamientos de 2850 y $2650 \mathrm{~kg} / \mathrm{ha}$ respectivamente. El testigo sin control sufrió una disminución del $35 \%$ respecto al testigo desmalezado manualmente cuyo rendimiento fue de $2920 \mathrm{~kg} / \mathrm{ha}$.
\end{abstract}

Palabras-claves: clorasulam-metil, clorimuron-etil, imazethapyr, lactofen, mezcla de herbicidas.

\section{ABSTRACT \\ Control of Euphorbia dentata in soybean with postemergence herbicides}

Euphorbia dentata is an annual weed which grows during spring and summer. Its has acquired importance mainly associated with soybean crops in the central area in Buenos Aires Province, Argentina. This weed is highly competitive in soybean fields and its control is very difficult. The objective of this work was to evaluate the effectiveness of Euphorbia dentata control with postemergence herbicides in soybean crops. Field studies were conducted in Azul Co., in a highly naturally infested soybean crop (110 plants of Euphorbia dentata $/ \mathrm{m}^{2}$ ). The treatments were: imazethapyr $100 \mathrm{~g} / \mathrm{ha}$, chlorasulam-methyl $33.6 \mathrm{~g} / \mathrm{ha}+$ no ionic surfactant $0.2 \% \mathrm{v} / \mathrm{v}$, lactofen $96 \mathrm{~g} / \mathrm{ha}+$ no ionic surfactant $0.2 \% \mathrm{v} / \mathrm{v}$, chlorimuron-ethyl $8.75 \mathrm{~g} / \mathrm{ha}+\mathrm{no}$ ionic surfactant $0.2 \% \mathrm{v} / \mathrm{v}$, chlorasulam-methyl $33.6 \mathrm{~g} / \mathrm{ha}+$ lactofen $96 \mathrm{~g} / \mathrm{ha}+$ no ionic surfactant $0.2 \% \mathrm{v} / \mathrm{v}$, chlorimuron-ethyl $8.75 \mathrm{~g} / \mathrm{ha}$ + lactofen $96 \mathrm{~g} / \mathrm{ha}+$ no ionic surfactant $0.2 \% \mathrm{v} / \mathrm{v}$, untreated control and control without weeds which were removed by hand. The experimental design was a randomised complete block with four replications. Evaluations were made 20 and 45 days after 
application and on harvest. No treatment reached $70 \%$ control. The highest levels of effectiveness were obtained with imazethapyr $(67 \%)$ and with the mixture chlorasulam+lactofen $(65 \%)$ with no significant differences between them. Soybean yield showed a similar response: the productivity in those treatments were 2850 and $2650 \mathrm{~kg} / \mathrm{ha}$ respectively. Crop productivity decrease by $35 \%$ in untreated control compared with the treatment without weeds whose yield was of $2920 \mathrm{~kg} / \mathrm{ha}$.

Key words: chlorasulam-methyl, chlorimuron-ethyl, imazethapyr, lactofen, mix tank.

\section{INTRODUCCIÓN}

Euphorbia dentata es una maleza anual, de ciclo primavero-estival, de amplia distribución en Norteamérica, en las zonas templadas del este de los Estados Unidos hasta Texas y México (Webster,1967).

En la República Argentina fue citada por primera vez por Parodi (1930) en Pergamino, al norte de la Provincia de Buenos Aires. En la zona Centro de la Provincia de Buenos Aires, es detectada a partir del año 1983, localizada en focos aislados asociada a los primeros cultivos de soja desarrollados en esa área. Actualmente, la misma se encuentra distribuida en el $85 \%$ del área agrícola del partido de Azul, con densidades que van desde 20 a 200 plantas $/ \mathrm{m}^{2}$ y se observa un crecimiento del área de infestación, presentándose como maleza en otras localidades cercanas como Tandil, Olavaria y Benito Juárez (Juan \& Saint-André 1995 ; Juan et al., 1996).

En ésta zona Euphorbia dentata es considerada una especie altamente competitiva y de difícil control. Si bien inicialmente se la caracterizó como maleza en el cultivo de soja, también se halla presente en girasol y maíz, e incluso en trigos de ciclo corto (de primavera) a partir del mes de octubre (Juan et al., 1996).

En la zona sojera central de la República Argentina (centro y sur de la provincia de Santa Fé, norte de la provincia de Buenos Aires y centro y sudeste de la provincia de Córdoba) es una maleza de importancia secundaria, debido a que se la encuentra presente sólo en un porcentaje bajo de los lotes, comparándola con otras especies (Giorda \& Baigorri, 1997), pero en el área de estudio se encuentran cada vez con mayor frecuencia stands puros de Euphorbia dentata a pesar de los tratamientos con herbicidas, lo que indica la dificultad que representa su control químico y la consecuente expansión del área ocupada por la misma.

Existen pocos antecedentes sobre Euphorbia dentata, pero la revisión bibliográfica indica que la especie tiene ciertas características que la asemejan con otra Euforbiácea anual ampliamente difundida como es Euphorbia heterophylla que es una de la principales malezas latifoliadas en cultivos de soja de diversos países del mundo (Nester et al., 1979; Harger $\&$ Nester, 1980; Wilson, 1981 ; Willard et al., 1994).

El objetivo de este trabajo fue evaluar la eficacia del control de la maleza Euphorbia dentata con herbicidas postemergentes en soja.

\section{MATERIAL Y MÉTODOS}

Los trabajos fueron llevados a cabo en condiciones de campo, durante las campañas 1997/98 y 1998/1999, en el partido de Azul, provincia de Buenos Aires, República Argentina. En un lote seleccionado por su historia de alta infestación de Euphorbia dentata, se implantó un cultivo de soja CV. AX 4422 (grupo IV) a $70 \mathrm{~cm}$ entre hileras, en parcelas experimentales de $6 \times 12 \mathrm{~m}$. El ensayo fue mantenido libre de otras especies de malezas latifoliadas desde la emergencia a través de desmalezado manual y de gramíneas por medio de una aplicación post-emergente de fluazifop-butil (Lee $5 \%$ Listo), a una dosis de $50 \mathrm{~g} /$ ha realizada 10 días antes que los tratamientos ensayados. Además, a través de raleo manual se uniformó la densidad de Euphorbia dentata dejando un stand homogéneo de 110 plantas $/ \mathrm{m}^{2}$.

Cuando la maleza alcanzó el estado de 4 hojas desarrolladas fueron realizados los siguientes tratamientos: A) imazethapyr $100 \mathrm{~g} /$ ha (LS 10\% Pivot H), B) clorasulam-metil $33,6 \mathrm{~g} / \mathrm{ha}$ (GD 84\% Pacto), C) lactofen $96 \mathrm{~g} / \mathrm{ha}$ (Lee $24 \% \mathrm{Co}-$ bra), D) clorimurón-etil 8,75 g/ha (GD 25\% Classic), E) clorasulam-metil 33,6 g/ha + lactofen $96 \mathrm{~g} / \mathrm{ha}$ (Pacto + Cobra), F) clorimurón-etil $8,75 \mathrm{~g} / \mathrm{ha}$, + lactofen $96 \mathrm{~g} / \mathrm{ha}$ (Classic + Cobra), testigo enmalezado y testigo limpio manualmente.

Las aplicaciones fueron realizadas con un equipo de aspersión con presión constante $\left(\mathrm{CO}_{2}\right)$ a $3 \mathrm{~kg} / \mathrm{cm}^{2}$ con un caudal equivalente a $150 \mathrm{l} / \mathrm{ha}$. Todos los tratamientos, excepto el imazethapyr, fueron realizados con el agregado de un coadyuvante no iónico al $0,2 \% \mathrm{v} / \mathrm{v}$ en la solución de aspersión.

Se realizaron evaluaciones de eficacia de control y fitotoxicidad a los 20 y 45 días desde la aplicación (DDA) y de los componentes del rendimiento y rendimiento final del cultivo a la cosecha. Se utilizó un diseño de bloques al azar con 4 repeticiones. Los datos se analizaron estadísticamente a través de análisis de varianza y las medias fueron comparadas por el test de Student-Newman-Keuls con $\mathrm{p} £ 0,05$.

\section{RESULTADOS Y DISCUSIÓN}

En la Figura 1, puede observarse que la eficacia de control evaluada a los 20 DDA resultó baja para todos los tratamientos utilizados, lográndose como máximo un $55 \%$ con 
la mezcla de clorasulam-metil+lactofén que no obstante no difirió significativamente de los herbicidas lactofén (50\%), imazethapyr (42\%) ni de la mezcla clorimurón-etil+lactofén $(42 \%)$.

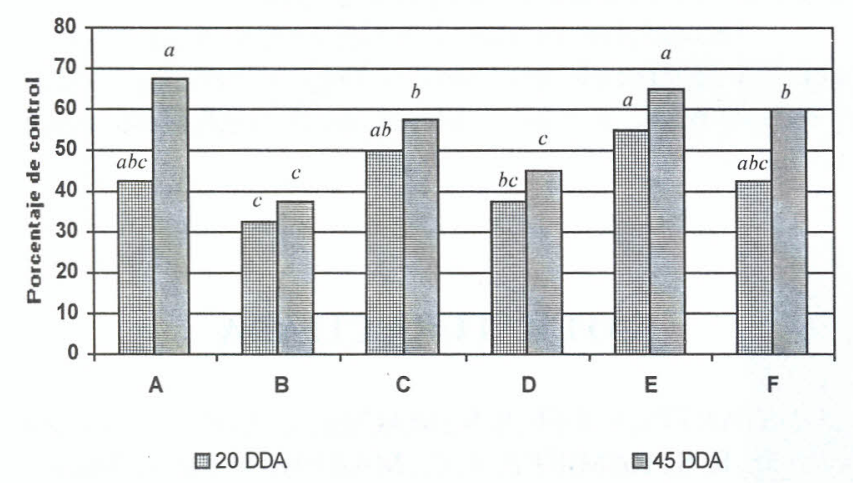

Figura 1. Eficacia de control de Euphorbia dentata a los 20 y 45 DDA. Azul, Pcia. de Buenos Aires, 1998.

Luego de 45 días, se observó que a pesar del mayor grado de control obtenido en general, ninguno de los tratamientos realizados alcanzó el $70 \%$ de efícacia. Los máximos niveles fueron obtenidos por imazethapyr (67\%) y la mezcla clorasulam-metil+lactofén (65\%), cuyos controles resultaron sustancialmente más altos con respecto a la primer evaluación realizada y no presentaron diferencias significativas entre sí.

Diversos autores han evaluado la mezcla de clorasulammetil+lactofén para el control de varias especies de malezas entre las que se encuentra Euphorbia heterophylla y particularmente para esta especie han logrado controles eficientes con dosis en general mayores a la utilizada en este ensayo, mencionando además algunos síntomas de fitotoxicidad temporario en el cultivo de soja pero sin incidencia sobre la productividad (Gelmini et al., 2000 ; Leite \& Almeida, 2000 ; Constantin et al., 2000). En nuestros ensayos no fueron apreciados síntomas de fitotoxicidad para ninguno de los herbicidas probados.

El nivel de control de Euphorbia dentata obtenido con imazethapyr fue similar a los reportados anteriormente por Juan \& Saint-André (1997), que determinan que la sensibilidad de esta especie a dicho herbicida está estrechamente relacionada con el momento de aplicación, obteniendo grados de control que oscilan entre el $80 \%$ cuando la misma se encuentra entre cotiledón a 2 hojas desarrolladas, $70 \%$ con la maleza entre 2 y 4 hojas desarrolladas y aproximadamente $50 \%$ cuando su estado fenológico fue mayor a 4 hojas y hasta primer ramificación.

La mezcla clorimurón-etil+lactofén, a pesar de lograr una eficacia cercana al $60 \%$, difirió de las anteriores y se comportó similar al tratamiento del herbicida lactofén aplicado individualmente. Los otros dos herbicidas, clorasulam-metily clorimurón-etil, cuando no fueron aplicados en mezcla con lactofén, mostraron niveles de control por debajo del $45 \%$ (Figura1).

Leite \& Almeida (2000) y Constantin et al. (2000) en sus reportes sobre el comportamiento de clorasulam-metil para el control de Euphorbia heterophylla, indican que cuando este herbicida se aplicó individualmente, logró resultados eficientes de control sólo con la mayor dosis probada que fue de $40 \mathrm{~g} / \mathrm{ha}$, mientras que en nuestros caso para el control de Euphorbia dentata se utilizó una única dosis de 33,6 g/ha que no logró una buena eficacia.

Para la especie Euphorbia heterophylla se menciona que la presencia de biotipos resistentes a herbicidas inhibidores de la enzima acetolactato sintetasa, es la causa de la baja eficiencia de control de herbicidas como imazethapyr, clorimurón-etil y clorasulam-metil cuando son aplicados individualmente (Gazziero et al., 1998 ; Vidal \& Merotto, 1999 ; Melhorança \& Pereira, 2000 ; Vargas et al., 2000 ; Da Silva et al., 2000). En el caso de Euphorbia dentata aún no se han detectado biotipos resistentes, pero como ya fue mencionado, se ha observado una gran influencia del estado fenológico sobre los resultados de control con imazethapyr que actualmente es el principal producto utilizado en los cultivos de soja en los que se presenta dicha especie.

Se presume que los niveles de control de alrededor del $50 \%$ obtenidos en la evaluación realizada a los 20 DDA para las mezclas de clorasulam-metil y clorimurón-etil con lactofén, han dependido principalmente de éste último herbicida que, por ser un difenil éter, es capaz de provocar una rápida acción de contacto que caracterizó la sintomatología observada inicialmente en estos tratamientos. En este sentido, durante la primer evaluación el herbicida lactofén, aplicado individualmente, obtuvo niveles de control similares a sus mezclas y teniendo en cuenta que en nuestro caso la aplicación se realizó cuando la maleza tenía 4 hojas desarrolladas, es posible que esto haya representado un estado fenológico relativamente avanzado, como para que un herbicida de dichas características pueda alcanzar controles superiores a los obtenidos.

En cuanto al rendimiento todas las diferencias detectadas se atribuyen a la densidad de Euphorbia dentata resultante de distintos niveles de control logrados por los tratamientos, se descarta la influencia de los herbicidas sobre la productividad del cultivo ya que, como fue mencionado anteriormente, a las dosis utilizadas no se registraron síntomas de fitotoxicidad en soja.

No se observaron diferencias significativas en la productividad del cultivo entre los tratamientos de imazethapyr y clorasulam-metil+lactofén, cuyos rendimientos fueron de 2850 y $2650 \mathrm{~kg} /$ ha respectivamente (Figura 2), no obstante el segundo difirió con el testigo desmalezado manualmente $(2920 \mathrm{~kg} / \mathrm{ha})$. Las densidad de 
Euphorbia dentata en el testigo sin control provocó una disminución del rendimiento de aproximadamente un 35\% respecto del testigo limpio, difiriendo significativamente de todos los tratamientos aplicados, aún de los que mostraron los más bajos niveles de control (clorasulammetil y clorimurón-etil).

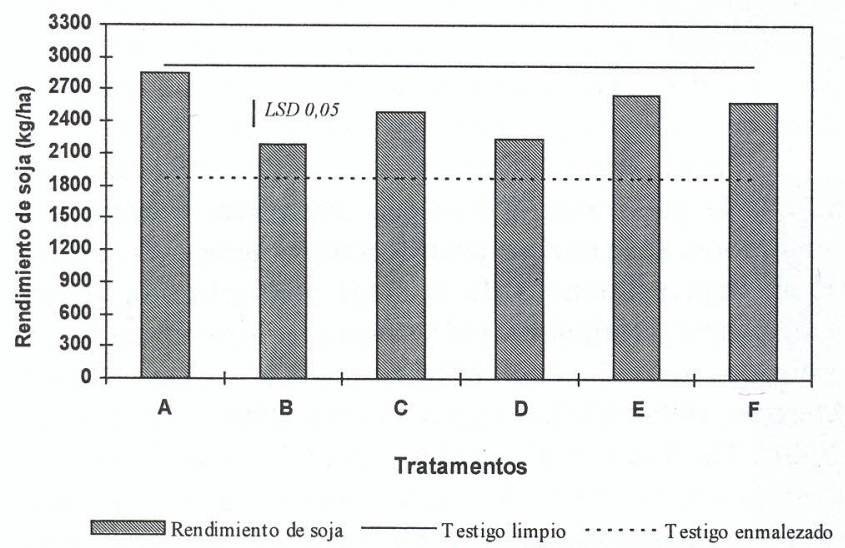

Figura 2. Rendimiento de soja a cosecha. Azul, Pcia. de Buenos Aires, 1998.

Con respecto a los componentes del rendimiento del cultivo (Tabla 1), el número de vainas por plantas resultó el más afectado. Los tratamientos de imazethapyr, lactofen y las mezclas de este último con clorasulam y clorimurón no difirieron significativamente respecto del testigo limpio y todos estos difirieron del testigo enmalezado. En cuanto al número de semillas por vaina, sólo difirieron del testigo enmalezado, el testigo limpio y el tratamiento con imazethapyr. No se observaron diferencias entre los tratamientos para el peso de 1000 semillas.

\section{CONCLUSIONES}

Ninguno de los herbicidas probados logró niveles comerciales de control de Euphorbia dentata, no obstante todos fueron capaces de disminuir los efectos competitivos que provoca la maleza en el cultivo de soja.

Imazethapyr resultó el tratamiento más adecuado ya que, si bien su grado de control no llegó al 70\%, fue el único tratamiento que no mostró diferencias en rendimiento con el testigo limpio.

\section{LITERATURA CITADA}

CONSTANTIN, J.; LEE, S. S.; MACIEL, C. D. G.; OLIVEIRA Jr., R. S.; RAMIREZ, A. C.; MARCHIORI Jr., O. Manejo de Euphorbia heterophylla na cultura da soja utilizando clorasulam-metil, isolado ou em mistura com outros herbicidas. In: CONGRESSO BRASILEIRO DAS CIENCIAS DAS PLANTAS DANINHAS, 22., Foz do Iguaçu, PR., 2000. Resumos... Londrina, PR: SBCPD, 2000. p. 147.

DA SILVA, A. A.; BOREM, A.; VARGAS, L. Chemical control of Euphorbia heterophylla L. biotypes resistant to acetolactate synthase (ALS/AHAS) inhibitors. In: INTERNATIONAL WEED SCIENCE CONGRESS, $3^{\text {th }}$., Foz do Iguassu, PR., 2000. Abstracts... Corvallis, Oregon: IWSS, 2000.p. 154.

GAZZIERO, D. L. P.; BRIGHENTI, A. M.; MACIEL, C. D. G.; CHRISTOFOLLETI, P. J.; ADEGAS, F. S.; VOLL, E. Resistência de amendoim-bravo aos herbicidas inibidores da enzima ALS. Planta Daninha, v. 16, n. 2, p. 117-125, 1998.

Tabla 1. Efecto de herbicidas sobre los componentes del rendimiento de soja. Azul, Pcia. de Buenos Aires, 1998.

\begin{tabular}{lccc}
\hline \multicolumn{1}{c}{ Tratamiento } & $\mathbf{N}^{\circ}$ vainas/planta & $\mathbf{N}^{\mathbf{0}}$ semillas/vaina & Peso de 1000 semillas (g) \\
\hline Imazethapyr & 26,6 & 2,21 & 147 \\
Clorasulam metil & 20,1 & 2,25 & 146 \\
Lactofen & 24,0 & 2,17 & 145 \\
Clorimuron etil & 21,0 & 2,21 & 145 \\
Clorasulam + lactofen & 24,8 & 2,18 & 148 \\
Clorimuron + lactofen & 24,1 & 2,23 & 145 \\
Testigo enmalezado & 18,0 & 2,13 & 145 \\
Testigo limpio & 27,1 & 2,22 & 147 \\
\hline LSD 0,05 & 5,8 & 0,06 & 4 \\
\hline
\end{tabular}


GELMINI, G. A.; DA COSTA, E. A. D.; MATALLO, M. B. M. Avaliação da mistura de lactofen e clorasulam-metil no controle de folhas largas na cultura da soja. In: CONGRESSO BRASILEIRO DAS CIENCIAS DAS PLANTAS DANINHAS, 22., Foz do Iguaçu, PR., 2000. Resumos... Londrina, PR: SBCPD, 2000. p. 142.

GIORDA, L. M.; BAIGORRI, H. E. J., eds. EI cultivo de la soja en Argentina. Ed. INTA: Centro Regional Córdoba, 1997. $448 \mathrm{p}$.

HARGER, T. R.; NESTER, P. R. Wild poinsettia: a major soybean weed. Louisiana Agric., v. 23, p. 4-7, 1980.

JUAN, V. F; SAINT-ANDRE, H. M. Comportamiento de Euphorbia dentata en la zona centro de la Provincia de Buenos Aires: biología de la germinación y sus efectos competitivos sobre el crecimiento de soja. In: CONGRESO LATINOAMERICANO DEMALEZAS, 12 ., Montevideo, Uruguay, 1995. Actas... Montevideo: INIA, Serie Técnica 56, 1995.p. 174-178.

JUAN, V. F.; SAINT-ANDRE, H. M. Control de lecherón (Euphorbia dentata) en soja con imazethapyr aplicado en distintos estados fenológicos. In: CONGRESO LATINOAMERICANO DE MALEZAS, 13., Buenos Aires, Argentina, 1997. Actas... Buenos Aires: ASAPROVE, 1997.v. 2, p. 128-133.

JUAN, V.F.; SAINT-ANDRE, H. M.; CARBONE, E.; ORFILA, E. N.; SCARAMUZZINO, R. L. Estudios sobre lecherón (Euphorbia dentata Michaux) en la zona centro de la provincia de Buenos Aires. Planta Daninha, v. 14, n. 2, p. 102-109, 1996.

LEITE, C. R. F.; ALMEIDA, J. C. V. Eficiência e seletividade agronômica do herbicida chlorasulam-methyl em posemergência na cultura da soja. In: CONGRESSO BRASILEIRO DAS CIENCIAS DAS PLANTAS DANINHAS,
22., Foz do Iguaçu, PR., 2000. Resumos... Londrina, PR: SBCPD, 2000.p. 141.

MELHORANÇA, A. L.; PEREIRA, F. D. A. R. Eficiencia do herbicida lactofen no controle de Euphorbia heterophylla resistente aos herbicidas inibidores da enzima acetolactato sintase. Revista Brasileira de Herbicidas, v. 1, n. 1, p. 53-60, 2000

NESTER, P. R.; HARGER, T. R.; McCORMICK, L. L. Weed watch-wild poinsettia. Weeds Today, v. 10, p. 25, 1979.

PARODI, L. R. Ensayo fitogeográfico sobre el partido de Pergamino. Revista de la Facultad de Agronomía y Veterinaria UBA, v. 8, n. 1, p. 218, 1930.

VARGAS, L.; DA SILVA, A. A.; DE REZENDE, S. T. Identification of Euphorbia heterophylla L. biotypes resistant to acetolactate synthase (ALS/AHAS) inhibitors. In: INTERNATIONAL WEED SCIENCE CONGRESS, $3^{\text {th }}$., Foz do Iguassu, PR., 2000. Abstracts... Corvallis, Oregon: IWSS, 2000. p. 139.

VIDAL, R. A.; MEROTTO Jr., A. Resistencia de amendoimbravo aos herbicidas inibidores da enzima acetolactato sintase. Planta Daninha, v. 17, n. 3, p. 367-373, 1999.

WEBSTER, G. L. The genera of euphorbiaceae in the southeastern United States. Journal Arnold Arbor. Harv. Sci., v. 48, n. 4, p. 363-420, 1967.

WILLARD, T. S.; GRIFFIN, L. J.; REYNOLDS, D. B.; SAXTON, A. M. Interference of wild poinsettia (Euphorbia heteropylla) with soybean (Glicine max). Weed Technology, v. 8, p. 679-683, 1994.

WILSON, A. K. Euphorbia heterophylla: a review of distribution, importance and control. Trop. Pest Management, v. 27, p. 32-38, 1981. 
